\title{
Positive kardiovaskuläre Endpunktstudien waren Highlights des vergangenen Jahres
}

\author{
Beim Diabetes-Update 2017 erhielten die Teilnehmer eine Zusammenfassung der wichtigen \\ neuen Erkenntnisse des vergangenen Jahres in der Diabetologie. Der Beleg des kardiovaskuläres \\ Schutzeffektes dreier Antidiabetika gehört sicher dazu.
}

Die Herz-Kreislauf-Sicherheit ist eine Forderung bei der Zulassung neuer Antidiabetika. Nachgewiesen werden kann sie über kardiovaskuläre Endpunktstudien. Eine der wichtigsten Studien des vergangenen Jahres in diesem Zusammenhang sei die LEADER-Studie, in der die kardiovaskuläre Sicherheit des lang wirksamen GLP-1-Rezeptor-Agonisten Liraglutid bei Typ-2-Diabetikern mit hohem kardiovaskulärem Risiko untersucht wurde, berichtete Privatdozent Dr. Michael Lehrke vom Universitätsklinikum Aachen.

9.340 Patienten mit Typ 2-Diabetes, 81,3\% davon mit kardiovaskulären Erkrankungen in der Vorgeschichte, wurden über eine mittlere Studiendauer von 3,8 Jahren mit Liraglutid (1,8 $\mathrm{mg} / \mathrm{Tag}$ ) oder Placebo (bei standard of care) behandelt [1]. Als Vorerkrankung hatten $31 \%$ einen Myokardinfarkt und $16 \%$ einen Schlaganfall erlitten, 24,7\% wiesen eine chronische Niereninsuffizienz und 17,1\% eine Herzinsuffizienz auf. Alle Patienten erhielten eine „standard of care“-Therapie (Statine: 72\%, ACEHemmer/ATR-Blocker: 83\%, Aspirin: 70\%, Metformin: 76\%, Sulfonylharnstoffe: $51 \%$, Insulin: $42 \%)$. Bei Studienbeginn betrug das LDL-C durchschnittlich $89,5 \mathrm{mg} / \mathrm{dl}$, der Blutdruck 136/77 mmHg und der $\mathrm{HbA}_{1 \mathrm{c}}$-Ausgangswert 8,7\%. Als primärer Endpunkt wurde eine Kombination aus kardiovaskulärem Tod, nicht tödlichem Herzinfarkt und nicht tödlichem Schlaganfall (3-Punkt MACE) untersucht.

Während der Studiendauer traten insgesamt 1.302 primäre Ereignisse auf. Eine Behandlung mit Liraglutid reduzierte den primären Endpunkt um 13 \% (608 von 4.668 Patienten [13,0\%] vs. 694 von 4.672 Patienten [14,9\%] unter Placebo (HR: 0,87; $95 \%$-KI: 0,78-0,97; $\mathrm{p}<0,001$ für nicht Unterlegenheit; $\mathrm{p}=0,01$ für Überlegenheit). Der Unterschied wurde wesentlich durch eine $22 \%$ ige Reduktion der kardiovaskulären Mortalität getrieben (Liraglutid 219 Ereignisse [4,7\%]; Placebo 278 Ereignisse [6,0\%]; HR: 0,78; $95 \%-K I: 0,66-0,93 ; p=0,007)$. Außerdem bestand ein deutlicher Trend für eine Reduktion nicht tödlicher Myokardinfarkte (n.s.), während sich die Häufigkeit nicht-tödlicher Schlaganfälle nicht relevant unterschied. Die Behandlung mit dem GLP-1-Rezeptor-Agonisten führte zu einer $15 \%$ igen $\mathrm{Re}$ duktion der Gesamtmortalität (Liraglutid 381 Ereignisse [8,2\%], Placebo 447 Ereignisse [9,6\%]; p=0,02). Für Revaskularisationsereignisse oder Hospitalisierung wegen Herzinsuffizienz bestand kein Unterschied.

In der Subgruppenanalyse profitierten Besonders Patienten mit bereits bestehenden kardiovaskulären Erkrankungen oder mit eingeschränkter Nierenfunktion. Bei Studienende wiesen mit Liraglutid behandelte Patienten im Vergleich zu Placebo einen $0,4 \%$ niedrigeres $\mathrm{HbA}_{1 \mathrm{c}}$ auf. Ebenso zeigten sich eine signi- fikante Gewichtsreduktion von $2 \mathrm{~kg}$ und eine Blutdruck-Reduktion um 1,2 mmHg. Liraglutid erhöhte im Vergleich zu Placebo die Herzfrequenz signifikant um 3 Schläge pro Minute.

Ebenso führte Liraglutid zu einer signifikanten Reduktion neu auftretender Nephropathien um $22 \%(\mathrm{p}=0,003)$ und es kam zu signifikant weniger schweren Hypoglykämien als unter Placebo $(2,4 \%$ vs. $3,3 \%, p=0,02)$. Im Liraglutid-Arm waren mehr Drop-outs aufgrund von Nebenwirkungen $\mathrm{zu}$ verzeichnen (9,5\% vs. 7,3\% unter Placebo, p<0,001). Wie Lehrke zusammenfasste, demonstrierte die große und mit hoher statistischer Sicherheit ausgestattete kardiovaskuläre Outcome-Studie die kardiovaskuläre Überlegenheit des lang wirksamen GLP-1-Rezeptor-Agonisten im Vergleich zu „standard of care“. Die Therapie reduziert die CV-Mortalität und die Gesamtsterblichkeit bei Patienten mit Diabetes und hohem kardiovaskulären Risiko. Ähnlich positive Ergebnisse hat auch die kleinere und kürzere Studie SUSTAIN 6 gezeigt [2], in der die kardiovaskuläre Sicherheit (3-Punkt MACE) des sehr lang wirksamen GLP-1-RezeptorAgonisten Semaglutid bei 3.297 Typ-2-Diabetikern mit hohem kardiovaskulären Risiko untersucht wurde. Im Vergleich zu einer „standard-of-care“-Medikation kam es unter der VerumBehandlung zu einer 39 \%igen Reduktion nicht tödlicher Schlaganfälle und zu einer tendenziellen Reduktion nicht tödlicher Myokardinfarkte, während kein Unterschied bei der kardiovaskulären Mortalität oder der Gesamtmortalität bestand.

\section{Durchbruch in der Diabetestherapie}

Damit habe mit den lang wirksamen GLP-1-Rezeptor-Agonisten neben den 2GLT2-Inhibitoren eine weitere Substanzklasse eine kardiovaskuläre Überlegenheit gezeigt. Beide Substanzgruppen reduzieren die Gesamtmortalität. Dies sollte in den Therapiealgorithmen Berücksichtigung finden, so Lehrke.

Dagmar Jäger-Becker

\section{Literatur \\ 1. Marso SP et al., NEJM 2016, Jul 28; 375(4):311-22 \\ 2. Marso SP et al., NEJM 2016, Nov 10; 375(19):1834-1844}

Quelle: DiabetesUpdate 17./18. März 2017 in Mainz 